\title{
Prognostic effect of allogeneic hematopoietic stem cell transplantation on first and non-first complete remission in acute myeloid leukemia
}

\author{
Ning Hu${ }^{1}$, Zhiheng Cheng ${ }^{2}$, Yifan Pang ${ }^{3}$, Hongmian Zhao ${ }^{1}$, Hui Ding ${ }^{1}$, Li Chen ${ }^{1}$, Qianyu Li ${ }^{1}$, Yu Han ${ }^{1}$, \\ Tong Qin ${ }^{1}$, Yifeng Dai ${ }^{2}$, Yijie Zhang ${ }^{4}$, Jinlong Shi ${ }^{5}$, Depei $\mathrm{Wu}^{6}$, Lin $\mathrm{Fu}^{1,7,8}$ \\ ${ }^{1}$ Department of Hematology, Huaihe Hospital of Henan University, Kaifeng 475000, China; ${ }^{2}$ Department of Pathology and Medical Biology, \\ University Medical Center Groningen, University of Groningen, Groningen, The Netherlands; ${ }^{3}$ Department of Medicine, William Beaumont \\ Hospital, Royal Oak, MI, USA; ${ }^{4}$ Department of Respiratory, Huaihe Hospital of Henan University, Kaifeng 475000, China; ${ }^{5}$ Department of \\ Biomedical Engineering, Chinese PLA General Hospital, Beijing 100853, China; ${ }^{6}$ Department of Hematology, The First Affiliated Hospital of \\ Soochow University, Suzhou 215006, China; ${ }^{7}$ Department of Hematology, ${ }^{8}$ Translational Medicine Center, The Second Affiliated Hospital of \\ Guangzhou Medical University, Guangzhou 510260, China \\ Correspondence to: Lin Fu, MD, PhD. Department of Hematology, The Second Affiliated Hospital of Guangzhou Medical University, Guangzhou \\ 510260, China. Email: fulin022@126.com.
}

Submitted Feb 14, 2019. Accepted for publication Aug 19, 2019.

doi: 10.21037/atm.2019.08.120

View this article at: http://dx.doi.org/10.21037/atm.2019.08.120

Allogeneic hematopoietic stem cell transplantation (allo-HSCT) is considered the cure for intermediate or adverse-risk acute myeloid leukemia (AML) (1). It offers patients longer overall survival (OS) (2-4). Unfortunately, human leukocyte antigen (HLA) matched related donor is unavailable in more than $70 \%$ patients. Some of these patients, may experience relapse and subsequently achieve CR (non-CR1) with treatment. Some non-CR1 patients eventually receive allo-HSCT. It is unclear whether alloHSCT will improve the survival of these patients.

We collected 53 AML patients from The Cancer Genome Atlas (TCGA) database (https://cancergenome.nih.gov/) to determine whether prognosis was different between CR1 and non-CR1 AML patients following allo-HSCT (5). All patients were between ages 22 and 69, registered between November 2001 and March 2010. Non-CR1 was defined as complete remission after two or more relapse treatments. These data were used to assess prognostic criteria according to the recent European Leukemia Net (ELN) classification (6). OS was the study endpoint and was defined as the time from diagnosis to death or censoring at the last follow-up.

Clinical characteristics of the CR1 group $(n=32)$ and the non-CR1 group $(\mathrm{n}=21)$ were summarized in Table 1 . The median age of the CR1 group was significantly older than non-CR1 group (57.5 vs. $48, \mathrm{P}=0.031$ ); the $\mathrm{CR} 1$ group also had more patients older than 60 years ( 23 vs. 3, $\mathrm{P}=0.014)$. There were more favorable-risk patients in the non-CR1 group $(\mathrm{P}<0.001)$, No significant differences was found in the type of donor between CR1 and non-CR1groups.

Kaplan-Meier survival curves showed that there was no significant difference in OS between the CR1 and nonCR1 groups (Figure 1). We also assessed the prognostic significance of clinical and molecular characteristic, including remission status, risk category, age, peripheral WBC count, FLT3-ITD, NPM1 and DNMT3A with multivariate analysis (Table 2). The results indicated that none of these factors had any impact on OS.

We included the clinical features with significant differences between the two groups in Table 1 and typical prognostic factors into a multivariate analysis, and found no significant difference in OS between the CR1 group and the non-CR1 group (Table 2).

In this study, we found that there was no prognostic difference between CR1 and non-CR1 patients following allo-HSCT. Allo-HSCT might weaken the adverse effect of relapse in non-CR1 patients and should still be offered once consequential remission was achieved. 
Table 1 Comparison of clinical characteristics of the CR1 and the non-CR1 group

\begin{tabular}{|c|c|c|c|c|}
\hline Characteristic & CR1 (n=32) & Non-CR1 $(n=21)$ & Statistics & $P$ value \\
\hline CR2 & $0(0.0)$ & $16(76.2)$ & $\chi^{2}=34.924^{\S}$ & 0 \\
\hline CR3 & $0(0.0)$ & $3(14.3)$ & $\chi^{2}=4.846^{\S}$ & 0.028 \\
\hline CR4 & $0(0.0)$ & $1(4.8)$ & $\chi^{2}=1.553^{\S}$ & 0.213 \\
\hline Age/years, median (range) & 57.5 [22-69] & 48 [23-65] & $\mathrm{U}=217.5^{\star}$ & 0.031 \\
\hline Age group/n (\%) & & & $\chi^{2}=6.044^{\S}$ & 0.014 \\
\hline$<60$ years & $19(59.4)$ & $19(90.5)$ & & \\
\hline$\geq 60$ years & $13(40.6)$ & $2(9.5)$ & & \\
\hline WBC count $/ \times 10^{9} / \mathrm{L}$, median (range) & $11.95(0.6-118.8)$ & $33.2(1.5-223.8)$ & $\mathrm{U}=231.5^{\star}$ & 0.057 \\
\hline$<40$ & $24(75.0)$ & $13(61.9)$ & $\chi^{2}=1.032^{\S}$ & 0.310 \\
\hline$\geq 40$ & $8(25.0)$ & $8(38.1)$ & & \\
\hline BM blasts/\%, median (range) & 64 [30-99] & 75 [34-97] & $\mathrm{U}=270^{\star}$ & 0.230 \\
\hline$<50$ & $7(21.9)$ & $3(14.3)$ & $\chi^{2}=0.477^{\S}$ & 0.490 \\
\hline$\geq 50$ & $25(78.1)$ & $18(85.7)$ & & \\
\hline \multicolumn{5}{|l|}{ FAB subtypes/n (\%) } \\
\hline M5 & $3(9.4)$ & $1(4.8)$ & $\chi^{2}=0.387^{\S}$ & 1 \\
\hline M6 & $1(3.1)$ & $0(0.0)$ & $\chi^{2}=0.669^{\S}$ & 1 \\
\hline M7 & $1(3.1)$ & $0(0.0)$ & $\chi^{2}=0.669^{\S}$ & 1 \\
\hline \multicolumn{5}{|l|}{ Risk category/n (\%) } \\
\hline Favorable & $0(0.0)$ & $8(38.1)$ & $\chi^{2}=14.358^{\S}$ & 0 \\
\hline Intermediate & $18(56.3)$ & $10(47.6)$ & $\chi^{2}=0.379^{\S}$ & 0.538 \\
\hline Adverse & $13(40.6)$ & 3 (14.3) & $\chi^{2}=4.174^{\S}$ & 0.066 \\
\hline ND & $1(3.1)$ & $0(0.0)$ & $\chi^{2}=0.669^{\S}$ & 1.000 \\
\hline \multicolumn{5}{|l|}{ HSCT } \\
\hline Haplo & $0(0.0)$ & $0(0.0)$ & & \\
\hline Sib allo & 19 (59.4) & $8(38.1)$ & $\chi^{2}=2.297^{\S}$ & 0.166 \\
\hline MUD & $13(41.6)$ & $13(61.9)$ & $\chi^{2}=2.297^{\S}$ & 0.166 \\
\hline
\end{tabular}

*, denotes Mann-Whitney U test; ${ }^{\S}$, denotes chi-square test. CR, complete remission; FAB, French-American-British; WBC, white blood cell; BM, bone marrow; PB, peripheral blood; HSCT, hematopoietic stem cell transplantation. 


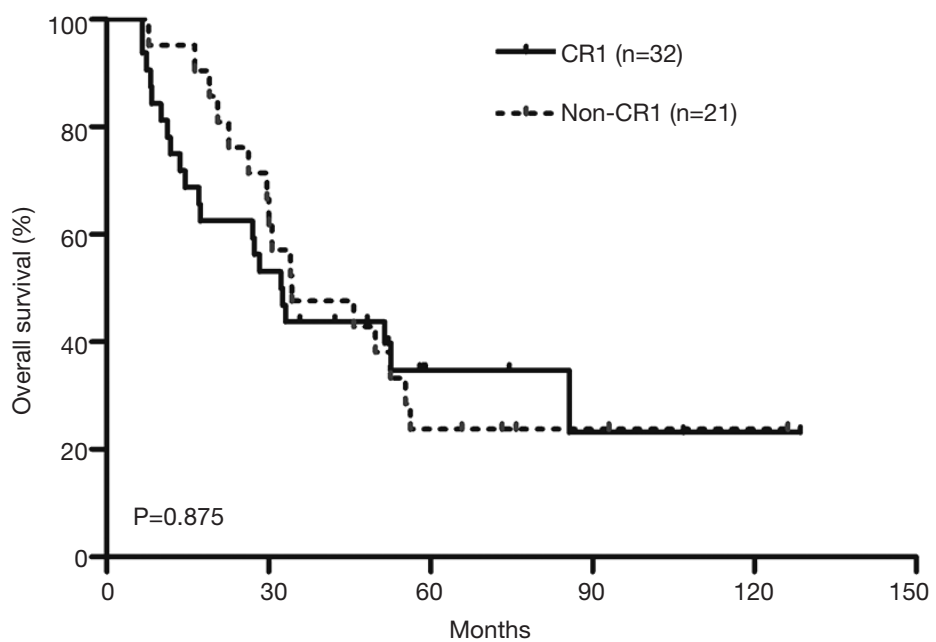

Figure 1 Kaplan-Meier curves of OS. No significant difference was found in OS between CR1 and non-CR1. P value was estimated by the log-rank test.

Table 2 Uni- and multivariate analyses for OS based on allo-HSCT

\begin{tabular}{|c|c|c|c|c|}
\hline Variables & \multicolumn{2}{|c|}{ Univariate analysis } & \multicolumn{2}{|c|}{ Multivariate analysis } \\
\hline Remission status (CR1 vs. non-CR1) & $1.053(0.549-2.021)$ & 0.876 & $0.751(0.33-1.804)$ & 0.522 \\
\hline Risk category (favorable/intermediate vs. adverse) & $1.291(0.634-2.629)$ & 0.482 & $0.613(0.251-1.499)$ & 0.283 \\
\hline Age (<60 vs. $\geq 60$ years) & $0.643(0.322-1.285)$ & 0.212 & $0.574(0.26-1.264)$ & 0.168 \\
\hline FLT3-ITD (positive vs. negative) & $0.686(0.312-1.508)$ & 0.348 & $0.575(0.200-1.648)$ & 0.303 \\
\hline NPM1 (mutated vs. wild type) & $0.919(0.418-2.020)$ & 0.834 & $1.265(0.429-3.736)$ & 0.670 \\
\hline DNMT3A (mutated vs. wild type) & $0.803(0.376-1.714)$ & 0.571 & $0.693(0.264-1.817)$ & 0.456 \\
\hline
\end{tabular}

OS, overall survival; CR, complete remission; WBC, white blood cell.

The optimum timing for allo-HSCT in AML patients remains controversial. A previous study found that $\mathrm{AML}$ patients who did not receive a transplant in CR1, but underwent allo-HSCT in CR2 had superior survival compared with the patients who did not underwent alloHSCT, particularly in the intermediate-risk patients (7). Compared with this study, our research found no difference in adult AML patients who underwent allo-HSCT in CR1 or subsequent CR, providing some insight into the timing to perform transplantation.

Our study was limited by its small sample size. The favorable risk patients are exclusively included in non
CR1 series because were considered for allogeneic HSCT only in case of AML relapse. Furthermore, the baseline characteristics of the CR1 group and the non-CR1 group were not balanced, with older patients in the CR1 group, and more good-risk patients in the non-CR1 group, which could have affected the results. Nevertheless, our study showed same prognosis in CR1 and non-CR1 patients following allo-HSCT, and supported that allo-HSCT was a reasonable post-remission therapy in adult AML patients who had reached another CR after relapse from CR1. Further studies with larger cohorts are warranted to validate our findings. 


\section{Acknowledgments}

Funding: This work was supported by grants from the National Natural Science Foundation of China (81500118, 61501519).

\section{Footnote}

Conflicts of Interest: The authors have no conflicts of interest to declare.

Ethical Statement: The authors are accountable for all aspects of the work in ensuring that questions related to the accuracy or integrity of any part of the work are appropriately investigated and resolved.

\section{References}

1. Kreso A, Dick JE. Evolution of the cancer stem cell model. Cell Stem Cell 2014;14:275-91.

2. Koreth J, Schlenk R, Kopecky KJ, et al. Allogeneic stem cell transplantation for acute myeloid leukemia in first complete remission: systematic review and meta-analysis

Cite this article as: Hu N, Cheng Z, Pang Y, Zhao H, Ding H, Chen L, Li Q, Han Y, Qin T, Dai Y, Zhang Y, Shi J, Wu D, Fu L. Prognostic effect of allogeneic hematopoietic stem cell transplantation on first and non-first complete remission in acute myeloid leukemia. Ann Transl Med 2019;7(18):500. doi: 10.21037/atm.2019.08.120 of prospective clinical trials. JAMA 2009;301:2349-61.

3. Cornelissen JJ, van Putten WL, Verdonck LF, et al. Results of a HOVON/SAKK donor versus no-donor analysis of myeloablative HLA-identical sibling stem cell transplantation in first remission acute myeloid leukemia in young and middle-aged adults: benefits for whom? Blood 2007;109:3658-66.

4. Kurosawa S, Yamaguchi T, Miyawaki S, et al. A Markov decision analysis of allogeneic hematopoietic cell transplantation versus chemotherapy in patients with acute myeloid leukemia in first remission. Blood 2011;117:2113-20.

5. Ley TJ, Miller C, Ding L, et al. Genomic and epigenomic landscapes of adult de novo acute myeloid leukemia. $\mathrm{N}$ Engl J Med 2013;368:2059-74.

6. Döhner H, Estey E, Grimwade D, et al. Diagnosis and management of AML in adults: 2017 ELN recommendations from an international expert panel. Blood 2017;129:424-47.

7. Burnett AK, Goldstone A, Hills RK, et al. Curability of patients with acute myeloid leukemia who did not undergo transplantation in first remission. J Clin Oncol 2013;31:1293-301. 